\title{
Ayurvedic management of Koshtashrita Kamala (Hepatocellular Jaundice) - A Case report
}

\author{
Case Report
}

\section{Ashwini C Amargol1*, Ashvini Kumar $\mathbf{M}^{2}$}

1. PG Scholar, 2. Professor and HOD, Department of Panchakarma, Sri Dharmasthala Manjunatheshwara College of Ayurveda and Hospital, Hassan, Karnataka, India.

\begin{abstract}
Kamala is a type of Liver disorder mentioned in our classics. It is caused by vitiation of pitta with the signs and symptoms like Haridra netra (yellow eyes), twak nakha (yellowish skin and nails) and Dourbalya (weakness), based on these clinical features, it can be well understood under the purview of Jaundice. The condition where yellowish discoloration of skin and sclera of the eyes due to high level of serum bilirubin is known as Jaundice. The pitta present in the body aggravates due to specific diet and life style, attains sthana samshraya in koshta due to kha vaigunya, further causes dagdhatwa of Rakta and Mamsa this condition is known as Koshtashirita Kamala. A case presented with Peeta Netrata, Peeta Varnyata Mutra, Aruchi, Agnimandhya etc was diagnosed as Koshtashrita Kamala it was intervened successfully with Shodhana and Shamana oushadi with proper pathya for a period of 11 days. The effect of treatment was assessed based on improvement in clinical signs and symptoms along with Liver Function Test. There was significant improvement in the condition by $60 \%$ thus Kamala can be well managed by Ayurvedic intervention.
\end{abstract}

Key Words: Ayurveda, Jaundice, Kamala, Liver, Pitta, Shodana.

\section{Introduction}

Yakrut (Liver) is one among the Koshtanga (organs of body) where the Bhutagni paka (metabolism at minutest level) takes place. It is the seat of Ranjaka pitta and mula (origin) for Raktavaha shrotas(1). Kamala is caused by pitta prakopakara ahara and vihara. The word 'Kamala' is composed of 2 words 'Ka'/Kaya (body) and 'Mala' (toxins) together forms 'Kamala', which signifies accumulation of toxins in the body. It is classified into 2 types - Koshtashrita Kamala (Hepatocellular jaundice) and Shakashrita Kamala (Obstructive Jaundice)(2). The pitta present in the body aggravates due to specific diet and life style attains vriddhavastha in koshta(3), this condition is named as Koshtashrita Kamala. Aggravated pitta causes dagdhatwa of Rakta and Mamsa(4) leading to Kamala. In Shakhashrita Kamala there is obstruction to the bile passage by Kapha(5).

Jaundice is a condition where there is yellowish discoloration of skin and sclera of the eyes due to high level of serum bilirubin in blood(6). In Hepatocellular Jaundice there will be increased conjugated serum bilirubin and increased urine bilirubin which comes under koshtagata Kamala. As the incidence of Koshtashrita Kamala is more, the disease is taken up

* Corresponding Author:

\section{Ashwini C Amargol}

PG scholar, Department of Panchakarma

Sri Dharmasthala Manjunatheshwara College of

Ayurveda and Hospital.

Hassan, Karnataka. India.

Email Id: ashwiniamargol14125@gmail.com for the study. Virechana is the first choice of treatment for pitta pradhana and rakta pradhoshaja vikara. Kamala is one such disorder mentioned in classics where mridhu virechana with tikta oushadhas(7) has prime role to play. When Ranjaka pitta gets sthanasamsraya in Urdhwajatru pradesha mainly in netra, jihwa and becomes stagnant even after the virechana karma, Nasya is indicated.

\section{Objectives}

To evaluate the Ayurvedic management of Koshtashrita Kamla.

\section{Materials and methods}

Medicine source- GMP certified company medicine from Sri Dharmasthala Manjunatheshwara College Pharmacy.

Subject source- IPD of Sri Dharmasthala Manjunatheshwara College of Ayurveda and Hospital, Hassan, Karnataka, India.

\section{$>$ Subjective Parameters}

1.Peeta Netra (yellowish discoloration of sclera)

2.Peeta Mutra (yellowish discoloration of urine)

3.Aruchi (Anorexia)

4.Agnimandya (loss of appetite)

5.Dourbalya (weakness)

6.Klama (giddiness)

7.Angamarda (bodyache)

8.Kandu (Itching)

- Normal 00

-Mild 01

-Moderate 02

-Severe 03 
Ashwini C Amargol et.al., Ayurvedic management of Koshtashrita Kamala (Hepatocellular Jaundice) - A Case report

$>$ Objective Parameters

1. Liver Function Test

\section{Case History}

A 70 yrs old male person came to OPD of Sri Dharmasthala Manjunatheshwara College of Ayurveda and Hospital, Hassan, Karnataka, India on 26/6/2020. The subject was apparently healthy 7 days back, gradually he developed Peeta Netrata, Peeta Varnyata Mutra, Aruchi, Agnimandhya, Dourbalyata, Klama, Angamardha and Kandu with these presenting complaints, he approached our institute for further management. So he was admitted under Panchakarma Department.

\section{History of Past Illness}

$\mathrm{K} / \mathrm{C} / \mathrm{O}$ Alcoholic since 45 years

$\mathrm{K} / \mathrm{C} / \mathrm{O}$ Type 2 Diabetic mellitus since 2 years on medication Tab Metformin 0-1-0 and

Tab Glimipride 1-0-1

$\mathrm{K} / \mathrm{C} / \mathrm{O}$ Hypertension since 2 years on medication $\mathrm{Tab}$ Amlong $5 \mathrm{mg}$ OD

\section{Physical examination}

General appearance - ill look

Built - moderate

Pallor - Absent

Icterus - Present in Sclera

Cyanosis - Absent

Clubbing - Present

Lympadenopathy - Absent

\section{Ashtavidha Pariksha}

Nadi (pulse) - Manda, Vata Pitta

Mala (Stool) - Asamyak pravruthi (irregular)

Mutra (urine) - Peeta varnyata (yellowish)

Jihwa (tongue) - Saama (coated)

Shabda (sound) - Prakruta (normal)

Sparsha (skin) - Sama shitoshna (normal temperature)

Drik ( eyes) - Netra peetata (yellowish sclera)

\section{Dashavidha Pareeksha}

$\begin{array}{ll}\text { - Prakriti } & \text { - Vata Pitta } \\ \text { - Sara } & \text { - Madhyama } \\ \text { - Samhanana } & \text { - Madhyama }\end{array}$ $\begin{array}{ll}\text { - Satva } & - \text { Madhyama } \\ \text { - Satmya } & - \text { Madhyama }\end{array}$

- Vyayama shakti - Alpa

- Aharashakthi -

- Abhyavaharana Shakti - Alpa

- Jarana Shakti - Alpa

- Vikruti

- Hetu - Madya

- Dosha - Pitta Kapha

- Dushya - Rasa, Rakta, Mamsa

- Desha - Jangala

- Kala - Vyaktavasta

- Vyadhibala - Madhyama

\section{Systemic Examination}

Central Nervous System - conscious and well oriented Respiratory System - Normal vesicular breath sound Cardiovascular System - S1 and S2 heard

Abdominal Examination -

- Inspection - normal shape, no scars, umbilicus inverted

- Palpation - tenderness positive over Right Hypochondrium, Epigastric, Right Lumbar and Iliac region

- Percussion - Tympanic Sound

- Auscultation - Bowel sound normal 16/min

\section{Nidhana Panchaka}

Nidhana - Madhya

Purvarupa - Klama, Aruchi

Rupa - Peeta Netrata, Peeta Varnyata Mutra, Aruchi, Agnimandhya, Dourbalyata, Klama, Angamardha and Kandu

Upashaya - Nothing significant

\section{Samprapti Ghataka}

\begin{tabular}{|c|c|}
\hline Dosha & Pitta \\
\hline Dushya & - Rasa, Rakta, Mamsa \\
\hline Ama & - Sama \\
\hline Agni & Mandagni \\
\hline Strotas & Rasavaha srotas, Raktavaha srota \\
\hline \multicolumn{2}{|c|}{ Annavaha srotas and Purishavaha srotas } \\
\hline Strotodushti & - Sanga, Vimargagamana \\
\hline Vyakthasthana & - Twak, Netra, Mutra \\
\hline Adishtana & - Yakrut \\
\hline Diagnosis & - Kosthashritha Kamala \\
\hline
\end{tabular}

\section{Treatment Schedule}

Table 1: Intervention planned with Shodhana and Shamana oushadhis

\begin{tabular}{|c|c|c|}
\hline Date & Intervention & Dose and Duration \\
\hline $26^{\text {th }}$ June Onwards & $\begin{array}{l}\text { Shirodhara with Dhanyamla } \\
\text { (room temperature) }\end{array}$ & 30 mins for 11 days \\
\hline $26^{\text {th }}$ June $-28^{\text {th }}$ June & Tab Kamadugha Mukta & 2 BD before food for 3 days \\
\hline \multirow{2}{*}{$\begin{array}{l}26^{\text {th }} \text { June } \\
\text { Onwards }\end{array}$} & Patolakaturohinyadi Kashaya & $\begin{array}{l}20 \mathrm{ml} \text { TID before food with equal quantity of warm } \\
\text { water }\end{array}$ \\
\hline & $\begin{array}{l}\text { Arogyavardhini Vati with } \\
\text { Nimba Kalka }\end{array}$ & $\begin{array}{c}2 \text { TID after food } \\
2 \mathrm{gm}\end{array}$ \\
\hline $26^{\text {th }}$ June- $1^{\text {st }}$ July & Avipattikara churna & $3 \mathrm{gm} \mathrm{BD}$ with warm water before food for 6 days \\
\hline
\end{tabular}


Combination of:

Avipattikara Churna (50gms) + Guduchi Churna

(20gms) + Pravala Bhasma (5gms)

$2^{\text {nd }}$ July - $7^{\text {th }}$ July

Avapeedana Nasya with Kumari swarasa

Netra Bhandana with Kumari pulp and Guduchi churna

2nd July

$4^{\text {th }}$ July

$6^{\text {th }}$ July
Avapeedaka Nasya with Jeemutaka Phala churna $120 \mathrm{mg}+$ Go Ksheera $3 \mathrm{ml}$ (6 bindu)
$5 \mathrm{gm} \mathrm{BD}$ with warm water before food

6 drops each nostril evening

30 minutes evening

3 bindu $(1.5 \mathrm{ml})$ in each nostril on empty stomach early in the morning (on alternate days)

\section{Diet Schedule}

Table 2: Pathya ahara niyama

\begin{tabular}{l|l|l|} 
Time Duration & Diet & Quantity \\
\hline 8 AM & Laja Manda & $200 \mathrm{ml}$ \\
\hline 11 AM & Yava Mantha & $200 \mathrm{ml}$ \\
\hline 2 PM & Peya & $250 \mathrm{ml}$ \\
\hline 5 PM & Shunti Kashaya & $200 \mathrm{ml}$ \\
\hline 8 PM & Peya & $200 \mathrm{ml}$ \\
\hline
\end{tabular}

Pathya - Boiled vegetables, Pomegranate

Apathya - Oily spicy food, non veg, curd, bakery food, day sleep and alcohol.

Subject was admitted for 11 days and found relief in generalized weakness, body pain and Icterus was reduced. Appetite and sleep were improved. There were improvements in both Subjective and Objective parameters from Moderate to Mild/Normal.

Table 3: Laboratory Investigations

\begin{tabular}{|c|c|c|c|c|}
\hline Date & $27 / 6 / 2020$ & $30 / 6 / 2020$ & $7 / 7 / 2020$ & $\begin{array}{l}\text { 11/8/2020 } \\
\text { (Follow } \\
\text { up) }\end{array}$ \\
\hline $\begin{array}{l}\text { Total } \\
\text { Bilirubin }\end{array}$ & $\begin{array}{l}14.7 \mathrm{mg} / \\
\mathrm{dl}\end{array}$ & $\begin{array}{l}16.8 \mathrm{mg} / \\
\mathrm{dl}\end{array}$ & $11 \mathrm{mg} / \mathrm{dl}$ & $2 \mathrm{mg} / \mathrm{dl}$ \\
\hline $\begin{array}{l}\text { Direct } \\
\text { Bilirubin }\end{array}$ & $9.6 \mathrm{mg} / \mathrm{dl}$ & $9.8 \mathrm{mg} / \mathrm{dl}$ & $6 \mathrm{mg} / \mathrm{dl}$ & \\
\hline $\begin{array}{l}\text { Indirect } \\
\text { Bilirubin }\end{array}$ & $5.1 \mathrm{mg} / \mathrm{dl}$ & $7 \mathrm{mg} / \mathrm{dl}$ & $5 \mathrm{mg} / \mathrm{dl}$ & \\
\hline $\begin{array}{l}\text { Total } \\
\text { Protien }\end{array}$ & $6.1 \mathrm{mg} / \mathrm{dl}$ & $5.1 \mathrm{mg} / \mathrm{dl}$ & $6.4 \mathrm{mg} / \mathrm{dl}$ & \\
\hline Albumin & $3.0 \mathrm{mg} / \mathrm{dl}$ & $2.9 \mathrm{mg} / \mathrm{dl}$ & $2.7 \mathrm{mg} / \mathrm{dl}$ & \\
\hline SGOT & $917 \mathrm{U} / \mathrm{L}$ & $1220 \mathrm{U} / \mathrm{L}$ & $351.7 \mathrm{U} / \mathrm{L}$ & \\
\hline SGPT & $728.1 \mathrm{U} / \mathrm{L}$ & $920 \mathrm{U} / \mathrm{L}$ & $278 \mathrm{U} / \mathrm{L}$ & \\
\hline ALT & 298 U/L & $205 \mathrm{U} / \mathrm{L}$ & $164 \mathrm{U} / \mathrm{L}$ & \\
\hline
\end{tabular}

Since the results were satisfactory along with improvement in the general condition of the subject, hence discharged and Shamana aushadi was prescribed for a period of 1 month as mentioned below-

Table 4: Discharge Medicine Medicine Dose

\begin{tabular}{l|l}
$\begin{array}{l}\text { Avipathikara Churna }(50 \mathrm{gms}) \\
\begin{array}{l}\text { Guduchi Churna }(50 \mathrm{gms}) \\
\text { Pravala Bhasma }(5 \mathrm{gms})\end{array}\end{array}$ & $\begin{array}{l}5 \mathrm{gms} \text { TID B/F with } \\
\text { warm water }\end{array}$ \\
$\begin{array}{l}\text { Patolakaturohinyadi Kashaya } \\
\text { Arogyavardhini Rasa }\end{array}$ & $\begin{array}{l}30 \mathrm{ml} \text { TID A/F with } \\
\text { warm water }\end{array}$ \\
\hline $\begin{array}{l}\text { TID B/F } \\
\text { Aloevera Juice }\end{array}$ & $15 \mathrm{ml} \mathrm{BD} \mathrm{B/F}$ with water \\
\hline
\end{tabular}

\section{Results and Discussion}

Kamala is a Pitta pradhana Tridhoshaja vyadhi, where Rakta and Mamsa dhatu are involved. Ishat sneha guna of pitta will be aggravated in the body, thus Rukshana with deepana will help to relieve the condition. Tikta, Kashaya and Madhura rasa Dravya helps in this perspective. Hence, Trivrut (Operculina terpethum (Linn.) Silva Manso), Patola (Tricosanthes dioica(Roxb)) and Katuki (Picrorhiza kurroa (Royle ex Benth)) are drug of choice for Ruksha virechana. These are the main ingredients in formulations such as Avipattikara churna, Patolakaturohinyadi Kashaya and Arogyavardhini Vati which have been administered in the above case. Even though Bahudoshavasta of Pitta is seen in Kamala, Tikshna Shodhana cannot be administrated due to Ojokshaya and Dourbalya. Hence, Acharya Vagbhata has advised to take out dhushiya pitta in Alpa Pramana but repeatedly. On this note, Avipattikara Churna is administered as Nitya virechaka (Cholegogue).

Pravala Bhasma (Coral calx) possess Kshariya and Madhura rasa, it is Laghu and sheeta guna. mitigates Tridosha, good appetizer and digestive stimulant. It also helps to improves physical strength and kills many infectious organisms in the body (8).

Guduchi (Tinospora cordifolia (Willd.) Miers) is Tikta Rasa Pradhana dravya(9), prevents fibrotic changes and promote regeneration of Parenchyma tissue. It has membrane stabilizing effect and modulation of kuffer cell activities(10).

Nasya-Ranjaka pitta gets sthanasamsraya in Urdhwajatru pradesha mainly in netra, jihwa and becomes stagnant even after the virechana karma, hence Nasya is indicated.

- Jeemuthaka Nasya - When Nasya was administered, intense yellow Rhinorrhoea was observed and patient had 3 episodes of vomiting on first day. There was sufficient reduction in Icterus.

- Kumari Swarasa Nasya- Kumari possess Tikta madhura rasa and sheeta virya(11).

Dhanyamla Shirodhara and Kumari Netra bandhana was effective in Anidrata.

Arogyavardhini vati contains Katuki as main ingredient which has tikta rasa and kaphapittahara karma and Purgative property(12). This formulation is considered as a potent digestive stimulant, anti inflammatory and anti viral property. Extremely effective in treating various liver problems like hepatitis, jaundice and fatty liver. Nimba (Azhadirachta Indica(A.Juss)) has Antioxidant, remove toxins and Anti viral(13). Kamadugha Muktha has sheeta veerya and Pittahara property(14). Patolakaturohinyadiyadi Kashaya acts as mrudu tikta virechaka( cholagogue), removes toxins from blood and liver, hepatoprotective. 


\section{Conclusion}

Koshtashrita Kamala is Pitta Pradhana Tridoshaja vyadhi, where Ojas Kshaya is also seen. Hence even though Virechana is a classical line of management for Prakupita Pitta, Tikshna Shodhana cannot be administered. Thus, it is successfully managed by Nitya Virechana, Nasya and other Pittahara Shamanaushadi along with proper pathya and Apathya.

\section{References}

1. Mahadevan L. Principles and Practice of Ayurvedic Clinical Medicine. First ed. Tamilnadu; Sharada Mahadeva Iyer Ayurvedic Educational and Charitable Trust; 2018. 151p.

2. Acharya Jadavaji Trikamji, Charaka samhita of Acharya Charaka. 2nd edition. Varanasi; Chaukamba Sanskrit Sansthana; 2015. 528p.

3. Acharya Jadavaji Trikamji, Charaka samhita of Acharya Charaka. 2nd edition. Varanasi; Chaukamba Sanskrit Sansthana; 2015. 528p.

4. Acharya Jadavaji Trikamji, Charaka samhita of Acharya Charaka. 2nd edition. Varanasi; Chaukamba Sanskrit Sansthana; 2015. 528p.

5. Acharya Jadavaji Trikamji, Charaka samhita of Acharya Charaka. 2nd edition. Varanasi; Chaukamba Sanskrit Sansthana; 2015. 532p.

6. Mahadevan L. Principles and Practice of Ayurvedic Clinical Medicine. First ed. Tamilnadu;Sharada
Mahadeva Iyer Ayurvedic Educational and Charitable Trust;2018.151p.

7. Acharya Jadavaji Trikamji, Charaka samhita of Acharya Charaka. $2^{\text {nd }}$ edition. Varanasi; Chaukamba Sanskrit Sansthana; 2015. 528p.

8. Angadi Ravindra. Text book of Rasashastra IATROChemistry and Ayurveda Pharmaceutics. Reprint ed. Varanasi; Chaukhamba subharati Prakashan;2018.442p.

9. Shastri J L N. Dravyaguna Vijnana. Reprint ed. Varanasi; Chaukhambha orientalia; 2012.41p.

10. Shastri J L N . Dravyaguna Vijnana. Reprint ed. Varanasi; Chaukhambha orientalia; 2012. 43p.

11. Shastri J L N . Dravyaguna Vijnana. Reprint ed. Varanasi; Chaukhambha orientalia; 2012.1050p.

12. Angadi Ravindra. Text book of Rasashastra IATROChemistry and Ayurveda Pharmaceutics. Reprint ed. Varanasi; Chaukhamba subharati Prakashan; 2018.582p.

13. Shastri J L N . Dravyaguna Vijnana. Reprint ed. Varanasi; Chaukhambha orientalia; 2012.127p.

14. Angadi Ravindra. Text book of Rasashastra IATROChemistry and Ayurveda Pharmaceutics. Reprint ed. Varanasi; Chaukhamba Subharati Prakashan; 2018.594p. 